\title{
Approaches to Support Differentiated Quality of Web Service
}

\author{
Sook-Hyun Ryu, Jae-Young Kim and James Won-Ki Hong \\ Department of Computer Science and Engineering \\ Pohang University of Science and Technology \\ Pohang, Korea \\ \{shryu, jay, jwkhong\}@postech.ac.kr
}

\begin{abstract}
The exponential rise in the number of Web users has inspired the creation of a diversity of Web applications. Hence, Web Quality of Service (QoS) is an increasingly critical issue in Web services, such as e-commerce, Web hosting, etc. In the future, improved QoS will be linked to a fee for service. Customers expect their requests to be served with a quality proportional to the amount charged to their accounts. Because most Web servers currently process requests on a first-come, first-serve basis, they do not provide differentiated QoS. This paper presents two approaches to implement differentiated quality of Web service. In the user-level approach, the Web server is modified to include a classification process, priority queues and a scheduler. However, with this approach, it is difficult to achieve portability. In this paper, a new, portable user-level approach is presented. In the kernel-level approach, a real-time scheduler to support prioritized user requests has been added to the operating system kernel. Prototype implementations for two approaches have been developed and their performances are evaluated.
\end{abstract}

\section{Introduction}

Today, most World Wide Web (WWW or Web) servers do not provide differentiated service quality to different requests of Web users. The Apache Web server [1], one of the most widely used Web servers, handles incoming requests on a first-come, firstserve basis. All requests correctly received are eventually handled, regardless of the type of request on the Web server. In this situation, premium users (i.e., those who pay more for higher quality of service) cannot be protected from overload in the Web server. Consequently, this general Web server does not provide differentiated QoS.

Recently, Web Quality of Service (QoS) becomes a critical issue in various Web services and has been studied in many ways $[2,3,4,5,6,7,8]$. With the term QoS, we refer to non-functional requirements, such as performance or availability requirements. QoS requirements are concerned with how an application or service will behave at run-time. QoS requirements may differ for different invocations of a service, based on diverse factors, such as user authentication or time of day.

In this paper, we propose two approaches; a user-level and a kernel-level, to provide differentiated QoS using priority-based scheduling. In the user-level approach, the Apache Web server is modified to include a classification process, priority queues 
and a scheduler. It is difficult to achieve portability when modifying a specific Web server. Thus, we propose a new user-level approach, which includes the classification and scheduling of user requests. In the kernel-level approach, a Linux kernel is extended to include a real-time scheduler to support prioritized Hypertext Transfer Protocol (HTTP) requests. We evaluate the performance of two prototypes and compare response times, throughput, and error rates for high and low priority requests.

The rest of this paper is organized as follows. Section 2 describes related work and Section 3 presents functional and non-functional requirements for design and implementation issues. Section 4 presents the system design architecture and classification approaches and then the performance evaluation results are explained in Section 5. Finally, Section 6 summarizes our work and discusses possible future work.

\section{Related Work}

A general architecture for Web server QoS was previously outlined in [2]. HP WebQoS is an enhancement to the HP-UX operating environment, providing a unique and advanced platform to assure high service quality for e-services on HP 9000 Enterprise Servers. HP's WebQoS stabilizes service delivery, assuring fast and consistent service quality to customers even under the competitive environment found on the Internet. WebQoS optimizes resources and permits developers to build more cost-effective solutions. WebQoS also enables businesses to prioritize service levels to allow higher service quality for the most important users and applications. The goal was to manage peaks in client request rates and to support differentiated QoS for users. Their solution essentially entails scheduling and admission control to improve the performance of high priority requests. Their architecture includes a management component so that configuration parameters can be remotely set and the server's operation monitored. This closely resembles our work. However, there are several differences. First, changes to the main Apache server code were required for both request classification and scheduling. Our new user-level prototype performs similar tasks without modifying the main Apache code. Second, they do not have the kernellevel approach concept.

Vasiliou et al. [3] presented the design of a QoS architecture that can be added to the Apache Web server to allow the server to provide a differentiated QoS. The QoS module can support changing scheduling algorithms and the values of parameters that are used by the scheduling algorithms. However, the concept of kernel-level approach was not considered. They proposed the services needed to provide a differentiated QoS to clients of a Web site, based on the client's identity and attributes. They also integrated an implementation of the services with the Apache Web server and described a service that can be used to create algorithms to suit a specific company's goal.

Almeida et al. [4] investigated a method to provide a differentiated QoS: prioritybased scheduling. The main metric for the QoS is latency in handling the HTTP request to the Web page. They showed both a user-level and a kernel-level approach. However, the user-level approach can be used only in a specific Web server, and this approach does not support portability. Scheduling policies can be preemptive or non- 
preemptive. They have implemented preemptive scheduling at the kernel-level, and non-preemptive scheduling at the user-level. Two important aspects of the scheduling policy must be mentioned. First, upon receiving a request, the scheduling policy must decide whether to process the request immediately, or to postpone the execution (sleep policy). Secondly, the scheduling policy must also decide when a postponed request must be allowed to continue (wakeup policy). This work allows a request to continue only in place of a completed request. When a request is completed, the scheduling policy must decide which of the postponed requests, if any, should be selected to execute in its place. If the policy allows lower priority requests to execute in the absence of higher priority requests, the scheduling policy is said to be workconserving. Otherwise, it is said to be non-work-conserving. A work-conserving policy tries not to allow processes to block while there are waiting requests. In this work, the Sleep and Wakeup policies have been implemented, by using thresholds for the maximum number of requests that can be concurrently handled at each priority level. Thus, a fixed number of slots exist for each priority level, and each incoming request must either occupy a slot (executes), or wait in a queue until it is allowed to execute (blocks). The related work has been compared and summarized in Table 1.

Table 1. Comparison of Related Work with Our Work

\begin{tabular}{|l|c|c|c|c|}
\hline & WebQoS (HP) & Vasiliou et al. & Almeida et al. & Our Work \\
\hline User-level approach & $\mathrm{O}$ & $\mathrm{O}$ & $\mathrm{O}$ & $\mathrm{O}$ \\
\hline Kernel-Level approach & $\mathrm{X}$ & $\mathrm{X}$ & $\mathrm{O}$ & $\mathrm{O}$ \\
\hline Portability & $\mathrm{O}$ & $\mathrm{X}$ & $\mathrm{X}$ & $\mathrm{O}$ \\
\hline Service levels & 3 & 2 & 2 & 2 \\
\hline URL classification & $\mathrm{O}$ & $\mathrm{O}$ & $\mathrm{O}$ & $\mathrm{O}$ \\
\hline Client IP classification & $\mathrm{O}$ & $\mathrm{O}$ & $\mathrm{X}$ & $\mathrm{O}$ \\
\hline $\begin{array}{l}\text { User authentication } \\
\text { classification }\end{array}$ & $\mathrm{X}$ & $\mathrm{X}$ & $\mathrm{X}$ & $\mathrm{O}$ \\
\hline
\end{tabular}

\section{Requirements}

In this section, we discuss requirements that must be considered during design and implementation. Requirements are divided into two parts - functional requirements and non-functional requirements.

\subsection{Functional Requirements}

In order to design and implement approaches to provide differentiated quality of Web service, certain functional requirements must be considered. The differentiated Web server requires the following functions: classification of requests, scheduling, and execution of requests.

The basic role of a general Web server is processing HTTP packets on a firstcome, first-serve basis. The general Web server listens to a signal which indicates that a connection has been made. After accepting the connection, the server must serve the user request. After serving the request, the server awaits the next signal. 
Unlike the general Web server, the differentiated Web server must provide differentiated QoS using priority-based scheduling. Therefore, we modify the general Web server into the differentiated Web server to add components that support differentiated QoS.

After accepting the connection, the differentiated Web server classifies the user request. The classification of requests is the first concern in the implementation of a differentiated Web server. The classification methods are as various as can be imagined. For example, in a server-based method, we can categorize a Universal Resource Locator (URL) required by the user. In a client-based method, we can classify the user request with the client IP address. After classification of the user request, the server must save it into a priority queue. A scheduler that employs a specific scheduling method assigns the user request in priority queues. The scheduling method is the second concern in implementing a differentiated Web server. As a specific scheduling method is applied, the throughput remains constant during peak demand. In addition, the error rate also remains constant during peak demand. After scheduling the user request, the server must serve the user request. Finally, this server is ready to receive another connection.

\subsection{Non-Functional Requirements}

To deliver differentiated QoS, a Web server can be modified to include a classification process, priority queues and a scheduler. However, portability is difficult to achieve when modifying a specific Web server. A module that supports a differentiated QoS must use a variety of general Web servers, such as Apache Web server and Microsoft's IIS Web server [9]. In addition, a differentiated Web server must be portable on various operating systems. Portability is the major concern among nonfunctional requirements.

General Web servers have evolved toward a multi-threaded architecture that either dedicates a separate thread to each incoming connection, or uses a thread pool to handle a set of connections with a smaller number of threads. Since a greater number of threads are used to handle user requests, more CPU capacity and memory usage is needed in general Web servers. Further, this condition is applied to a differentiated Web server equally. Because modules are added to support differentiated QoS, more CPU capacity and memory usage is needed in a differentiated Web server. Most Web servers do not serve every user request during peak demand or run short of CPU capacity and memory volume. If both Web servers have an equal CPU capacity and memory volume, the differentiated Web server must use as little CPU and memory usage as possible. Therefore, resource requirements are a secondary concern in nonfunctional requirements.

\section{Design of Two Approaches}

In this section, we discuss two approaches towards differentiated QoS in Web services, and present a differentiated Web server architecture and process structures. 


\subsection{User-Level Approach}

Here, we present a user-level approach to support differentiated quality of Web service. In the user-level approach, the general Web server, such as Apache Web server, is only modified to include components for supporting differentiated QoS. These components consist of connection and classification processes, priority queues, a schedule process, and execution processes, as illustrated in Fig. 1. Such components as classification processes, priority queues and the schedule process do not exist in a general Web server.

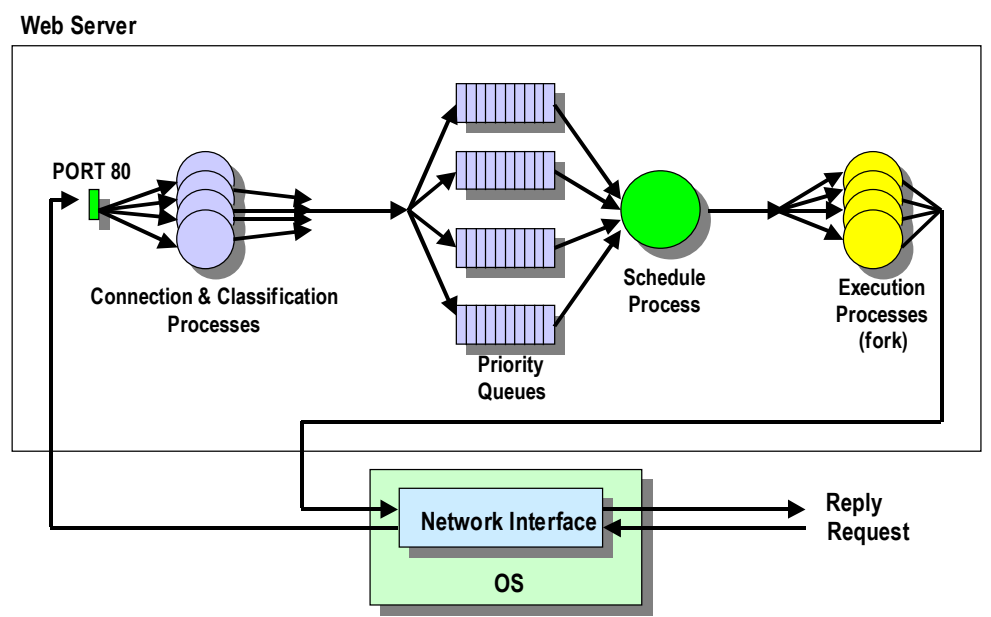

Fig. 1. User-Level Approach

First, incoming user requests from the network interface in the operating system are received through port 80 by connection and classification processes. Port 80 is a well-known port for supporting Web service. These processes classify the requests and place the requests on the appropriate priority queues. Several methods are used to classify requests. These classification mechanisms can be divided into two categories, a server-based and a client-based approach. In a later section, we explain the classification method in detail.

The number of priority queues implies the number of differentiation levels. A priority level is assigned for each priority queue. After requests are classified, the server must realize different service levels for each class of requests. This is accomplished by selecting the order of request execution.

A schedule process selects the next request, based on the scheduling policy. For example, requests from the highest priority queue will be processed first. Execution processes forked by the schedule process may be able to execute requests from any class, and will run until completion. Finally, execution results are sent to the user through a network interface.

Since we must modify a general Web server source code to support differentiated QoS in this approach, the system using this approach cannot handle the large variety of general Web servers. Because of this, we propose a new user-level approach to 
support portability, as follows.

\subsection{A New User-Level Approach for Supporting Portability}

Since the user-level approach described in Section 4.1 can be used only in a specific Web server, this approach does not support portability. For this reason, we propose a new user-level approach to support portability.

The new user-level approach consists of a differentiate module and a general Web server, as illustrated in Fig. 2 The differentiate module is an independent program for the realization of a differentiated QoS. The components of the differentiate module are similar to that of the user-level approach described in Section 4.1. However, we do not the general Web server, such as the Apache Web server.

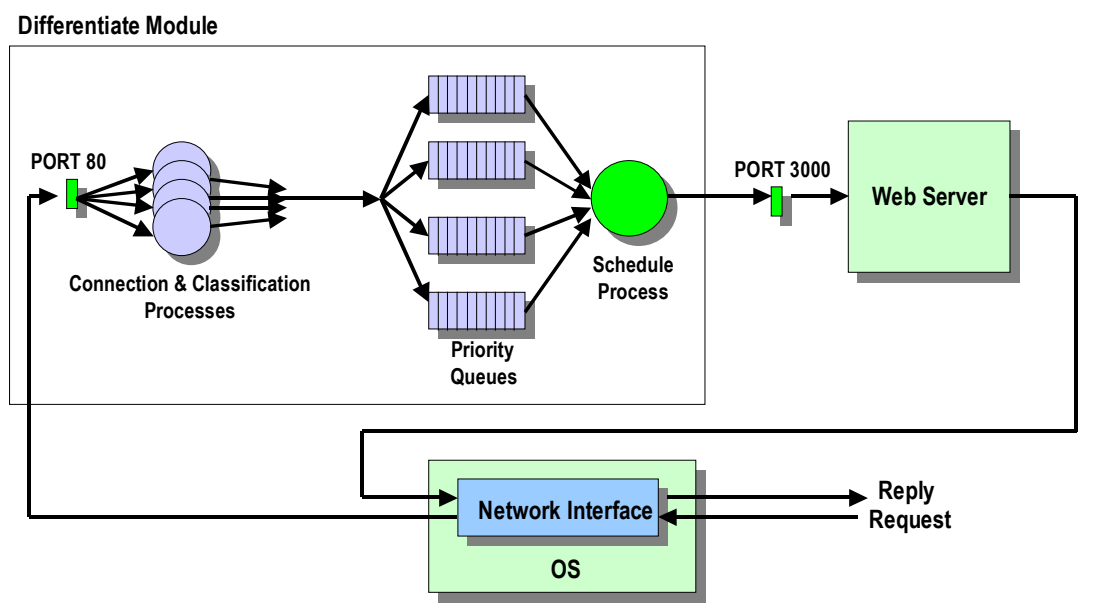

Fig. 2. New User-Level Approach

A schedule process selects the next request based on the scheduling policy. A selected request by a schedule process is sent to a general Web server through a specific port, such as 3000 . To accomplish this, the Web service port in a general Web server is configured into a specific port number, except for port 80 . The selected request is processed in a general Web server. Finally, the execution result is sent to the user through a network interface.

\subsection{Kernel-Level Approach}

We considered it heuristically advisable to attempt a kernel-level approach, since processes which act directly on the priorities assigned to the HTTP request might be more effective in controlling executions. Therefore, we use direct mapping from the user-level request priority to a kernel-level process priority. The kernel-level approach is based on the instrumentation of both a general Web server modification and an operating system with a real-time module, as illustrated in Fig. 3. 


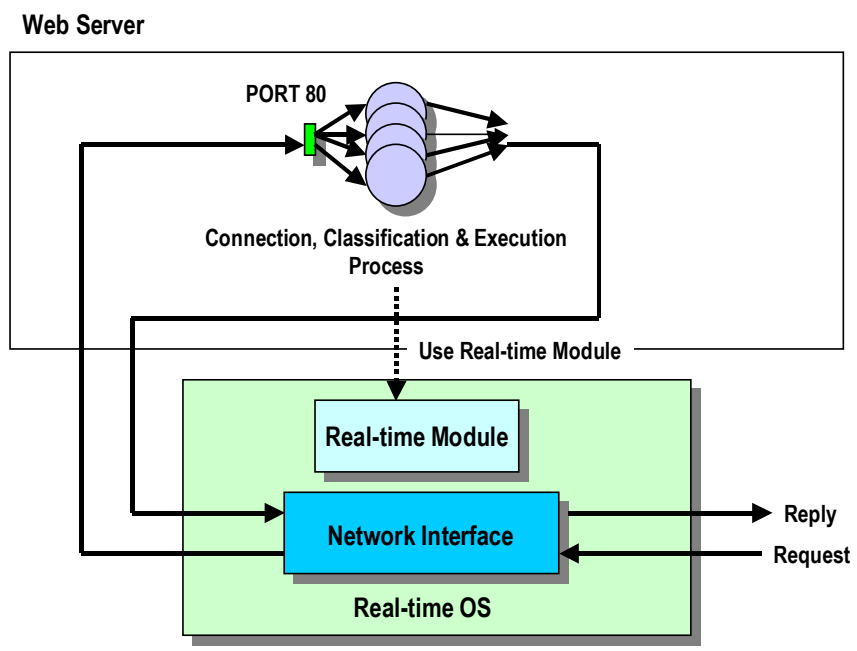

Fig. 3. Kernel-Level Approach

This approach to support differentiated QoS consists of a modification of a Web server to support request classification and an operating system with a real-time module. A general Web server is modified to have each HTTP process call the kernel to record the priority of the request currently being handled. The kernel is responsible for mapping this priority into the process priority. The kernel scheduler decides which process should use the CPU next. The kernel must keep track of all processes currently using the priority, along with their current state.

\subsection{Classification Methods}

A key requirement to support differentiated QoS is the ability to identify and classify the incoming requests according to each service class. Classification mechanisms can be divided into two general categories: a server-based and a client-based. The serverbased method classifies requests according to the contents or destination of the request. The client-based method characterizes requests according to the source of the request.

In the server-based method, the contents of URL of Web requests are used for determining a service level. A URL consists of a protocol header, a server address, a directory name, a file name and an extension. This information can be used to classify the relative importance of the request. Contents can be classified into different priority levels. Destination IP addresses can be used by a server when the server supports cohosting of multiple destinations (Web sites) on the same node. The server-based method is useful when performance of accessing some part of Web contents in a Web server needs to be protected and isolated from requests to other parts. Typical examples of this content discrimination can be found at various E-commerce sites. Web pages for processing customers' orders are more important and should be responded more reliable than pages for viewing a catalog of products. Further, users 
do not need any additional processing overhead or advance knowledge of the method.

In the client-based method, there are two kinds of choices for classification. The client's IP address is used to distinguish privileged clients from the non-privileged. This method is the simplest one to implement. However, since the client's IP address might be changed due to proxies or firewalls, this method has limitations. User authentication with username and password is another method used to classify a request to overcome the limitations. Both methods require advance agreements between the differentiated Web server and clients for the service level and quality. However, the client-based methods are able to provide fine-grained Web QoS for different individual users since the Web server can distinguish one client from another. This kind of differentiation is useful in enterprise environments where different users have different roles and priorities.

\subsection{Priority-Driven Scheduling Methods in the User-Level Approach}

After the requests are classified according to one of the above-mentioned classification schemes and admitted by the classifier, the server must actually realize different service levels for each class of requests. This is done by selecting the order of request execution. Execution processes are autonomous and select requests to process based on the scheduling policy. The scheduling policy may depend on queue lengths. Execution processes may be able to execute requests from any class. Alternatively, to reserve a capacity for higher-class processes, they may be restricted to executing higher-class traffic. The following presents several possible policy guidelines.

- Strict priority - This policy schedules all higher-class requests before lowerclass requests, even when low-priority requests are waiting.

- Weighted priority - This policy schedules a class based on its weight importance. For example, one class will receive twice as many scheduled requests if its class weight is twice that of another.

- Shared capacity - This policy schedules each class to a set capacity and any unused capacity can be given to another class. The class may also have a minimum reserve capacity that cannot be assigned to another class.

- Fixed capacity - This policy schedules each class to a fixed capacity that cannot be shared with another class.

- Earliest deadline first - This policy schedules based on the deadline for completion of each request. This can be used to provide a guaranteed predicted response time.

In our work, we use a strict priority scheduling method to provide differentiated Web service because it is very simple and requires less CPU capacity and memory volume than other priority scheduling methods. This condition is sufficient for nonfunctional requirements. If we use other priority scheduling methods, an additional resource management mechanism will be required. 


\section{Prototypes and Performance Comparison}

In this section two kinds of prototype implementation details are described and performance comparison between two prototypes with various request loads.

\subsection{Development Environment}

We have implemented both approaches on the development environment, as summarized in Table 2 Obviously, the development environment of the kernel-level approach is similar to that of new user-level approach, except that it uses the Montavista real-time scheduler [10] to support process priority on the operating system kernel level. The Montavista real-time scheduler is a specific scheduler to support a soft real-time kernel.

Table 2. Development Environment

\begin{tabular}{|c|c|c|}
\hline & New user-level approach & Kernel-level approach \\
\hline OS & Linux kernel 2.2.14 & Linux kernel 2.2.14 \\
\hline Realtime kernel & None & $\begin{array}{c}\text { Soft realtime kernel } \\
\text { (Montavista scheduler) }\end{array}$ \\
\hline Web server & Apache 1.3.12 & Apache 1.3.12 \\
\hline Language & C, PHP & C, PHP \\
\hline
\end{tabular}

We have implemented prototypes of these approaches on the Linux kernel 2.2.14 and the Apache Web server 1.3.12. We used C language for programming the prototypes of these approaches. Further, PHP [11] script was used to configure both the classification policy and user information in these prototypes.

\subsection{Performance Evaluation}

We evaluate the performance of two prototypes. We compare response times, throughput, and error rates for premium and basic client running with priority scheduling. The performance measurements are for high and low priority clients that monotonically increase with each client issuing an equal number of requests. Also, these clients are configured to issue requests simultaneously. Recall that the performance metrics are the throughput, the response time and the error rate of a request as perceived by the server. The proportion of high to low priority requests is $1: 1$. In Fig. 6, 7 and 8, the high priority requests have a better throughput, response time and somewhat better error rates in both user-level and kernel-level approaches.

In Fig. 6, we show the throughput when we vary the request rate for the user-level and kernel-level approaches. As the offered load increases, reply rate increases linearly until request rate reaches about 90 requests/s in the high priority requests using kernel-level approach. Further, if the request rate is over 90 requests/s, the reply 
rate is nearly fixed at 90 replies/s. However, as seen in Fig. 6, reply rate increases linearly until request rate reaches approximately 75 requests/s in the high priority requests using a user-level approach. If the request rate exceeds 75 requests/s, the reply rate is nearly fixed at 75 replies/s. This status occurs in low priority requests using both user-level and kernel-level approaches, too. For the most part, the throughput for requests using the kernel-level approach is greater than for requests using user-level approach in both high and low priory requests.

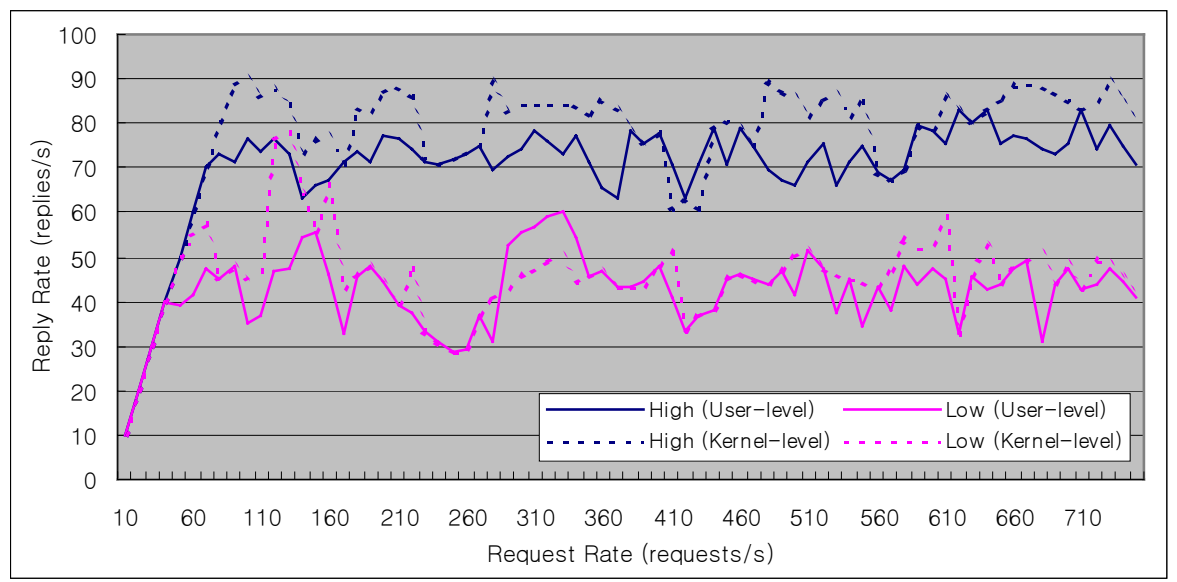

Fig. 6. Throughput in Differentiated Web Server

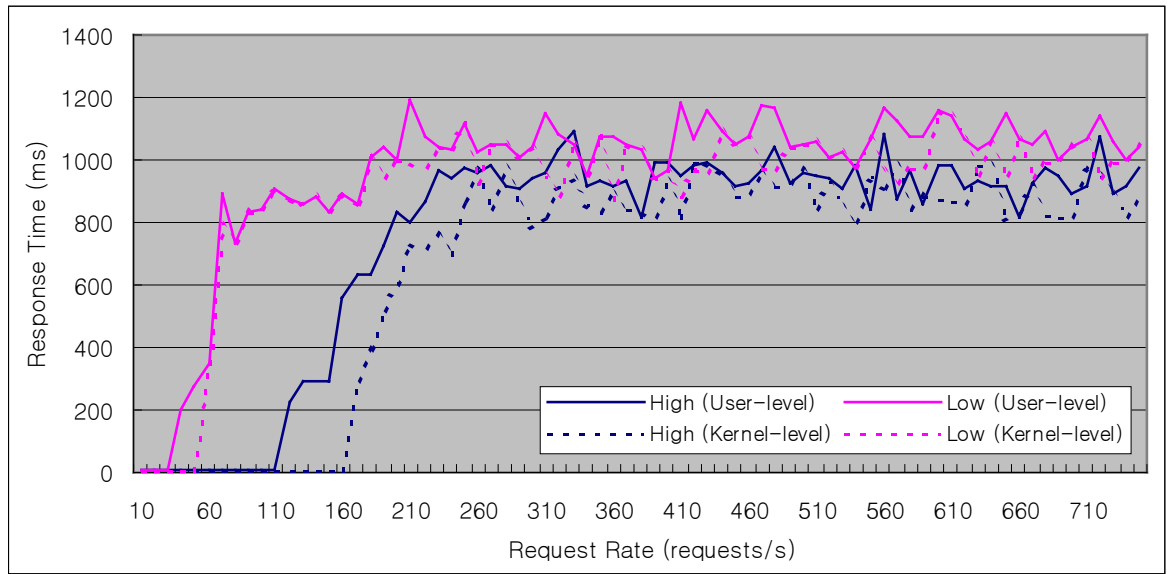

Fig. 7. Response Time in Differentiated Web Server

The response time is optimistic since it does not include requests which are rejected or which encounter an error. The second graph, Fig. 7, plots the average response time in milliseconds for all client requests completed successfully as a function of the total offered client demand rate. The differentiated Web server which 
uses the user-level approach is longer than one which uses the kernel-level approach in the response time. A new module for supporting portability in the differentiated Web server causes many processing delays.

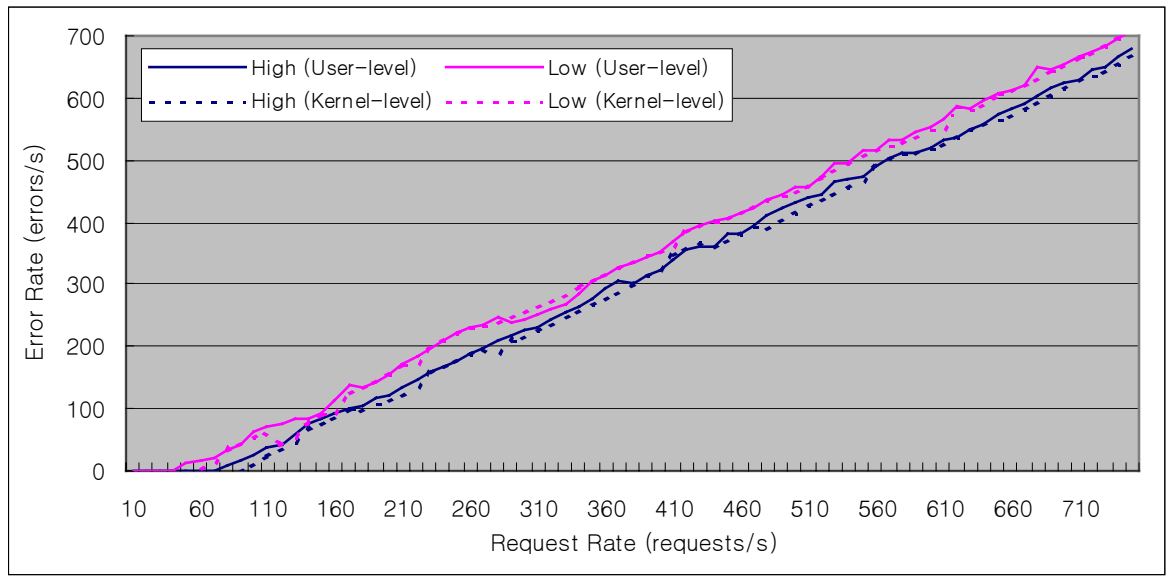

Fig. 8. Error Rate in Differentiated Web Server

Finally, the third graph, Fig. 8, plots client requests that encounter an error as a function of the total offered client demand rate in both the user-level and the kernellevel approaches. The error rate for requests using user-level approach is nearly equal to one using kernel-level approach in both high-level and low-level priority requests.

Overall, a differentiated Web server that applies a user-level approach becomes lower than one using a kernel-level approach in performance. However, supporting portability in such a user-level approach is an important factor. Most Web server administrators wish that the facilities to support differentiated service are applicable to various Web server and operating systems. It is likely that, with the increasing complexity of Web page design, the differentiation between high and low priority requests will increase as well. In the performance evaluation of the differentiated Web server, the responses of high priority requests are occasionally slower than one of low priority requests. In our view, the drop rate of low priority requests will be faster than one of high priority requests. In this situation, the length of the low priority queue will be temporarily shorter than that of the high priority queue. So this problem results. Future work will be devoted to ascertaining the exact cause of the problem.

\section{Conclusion and Future Work}

In this paper, we presented two approaches to support Web Quality of Service (QoS) that allows a general Web server to provide a differentiated QoS. Our methods categorize HTTP requests into classes based on classification methods, with the requests of each class handled differently by the desired scheduling method.

High priority clients are given better throughput, response time, and slightly better error rates. Supporting portability in that using user-level approach is considered 
factor as important. Most Web server administrators intend to implement a facility for supporting differentiated service in a variety of Web servers and operating systems.

We evaluate the performance of our implementations. They include analyzing errors, throughput, and response time of a server that handles one high priority client and one low priority clients with request rates that monotonically increase. This experiment models a situation where there are a various number of clients with subscriptions for high and low quality services. Further, we are making comparative performance evaluations of two approaches supporting differentiated quality of Web services.

More work is needed on scheduling algorithms. Many approaches can be taken to perform the scheduling of requests. The open-queue system of the Web will require complex algorithms to balance processing of requests in each class while maximizing system utilization. Designing a Web server framework to support server QoS is also a complex task. The Web server framework consists of an admission controller, a resource manager, a disk scheduler and a request scheduler. More work is also needed to integrate a server QoS with a network QoS. It is necessary to map their QoS parameters. Finally, we will extend current work on the Web server to other Internet servers, such as FTP server, VOD server, RealAudio server, and so on.

\section{References}

1. Apache HTTP Server Project Homepage, http://httpd.apache.org.

2. N. Bhatti and R. Friedrich., "Web Server Support for Tiered Services," IEEE Network, September/October 1999, pp. 64-71.

3. N. Vasiliou and H. Lutfiyya., "Providing a Differentiated Quality of Service in a World Wide Web Server," Proc. of the Performance and Architecture of Web Servers Workshop, Santa Clara, California USA, June 2000, pp. 14-20.

4. J. Almeida, M. Dabu, A. Manikutty, and P. Cai., "Providing Differentiated Levels of Service in Web Content Hosting," Proc. of the Workshop on Internet Server Performance, Madison, Wisconsin USA, March 1998, pp. 91-102.

5. M. Banatre, V. Issarny, F. Leleu, and B. Charpiot., "Providing Quality of Service over the Web: A Newspaper-based Approach," Proc. of the Sixth International World Wide Web Conference, Santa Clara, California USA, April 1997, Paper 149-Tec 110.

6. K. Beyer, M. Livny, and R. Ramakrishnan., "Protecting the Quality of Service of Existing Information Systems," Proc. of the Cooperative Information Systems, 1998, pp. 74-83.

7. R. Pandey, J. Barnes, and R. Olsson., "Supporting Quality of Service in HTTP Servers," Proc. of the SIGACT-SIGOPS Symposium on Principles of Distributed Computing, Puerto Vallarta, Mexico, June 1998, pp. 247-256.

8. T. Abdelzaher and N. Bhatti, "Web Server QoS Management by Adaptive Content Delivery," Proc. of the $7^{\text {th }}$ International Workshop on Quality of Service, London, England, June 1999, pp. 216-225.

9. Microsoft TechNet, http://www.microsoft.com/technet/iis.

10. Montavista Software, http:/www.montavista.com.

11. PHP Project Homepage, http://www.php.net 